\title{
A Practical Guide to Mitigate the Response of Corona Virus Pandemic in Radiotherapy Treatment
}

\author{
Shashank Bansal ${ }^{1}$, Vikas Jagtap ${ }^{2}$, Ghritashee Bora ${ }^{1}$
}

${ }^{1}$ Department of Radiation Oncology, Dr. B Borooah Cancer Institute, Guwahati, Assam, India. ${ }^{2}$ Department of Radiation Oncology, NEIGHRIMS, Shillong, Meghalaya, India.

\begin{abstract}
The current global pandemic of Corona virus has impacted the health care severely. Many of the non-emergency cases and general illness cases have been asked to avoid hospital visit or wait for elective surgeries. The same may not be possible for the patients suffering from cancer as it considered a major health problem owing to the risk of metastasis as well as disease becoming unresectable with time. Unfortunately, we don't have studies weighing the risk versus benefit ratio of delaying oncological treatment weighted against an added comorbidity of COVID infection. Radiotherapy being an integral part of cancer care is affected the most as it is delivered, continuous over a long period of time and unplanned gap because of travel restriction on patients makes decision making more difficult for radiation oncologist. In this article we've highlighted few major problems in delivering radiation to cancer patients at this time of global pandemic of corona virus and tried to find out possible solution.
\end{abstract}

Keywords: Radiotherapy- COVID-19- corona virus

\section{Introduction}

In December, 2019, a series of pneumonia cases of unknown cause emerged in Wuhan, Hubei, China, with clinical presentations greatly resembling viral pneumonia [1]. Deep sequencing analysis from lower respiratory tract samples indicated a novel coronavirus, which was named 2019 novel coronavirus (2019-nCoV). On January 30, the World Health Organization has declared the coronavirus outbreak a global Public health emergency. Till date more than ten lakh people have been infected worldwide and more than fifty thousand lost their lives because of this viral infection [2]. Common symptoms of COVID-19 are, fever, cough, myalgia or fatigue, less common features are, sputum production, headache, hemoptysis and diarrhea [3]. The disease can spread from person to person through small droplets from the nose or mouth which spreads when a person with COVID-19 coughs or exhales, hence social distancing and avoiding social gathering may protect us from acquiring this infection [4]. Self-initiated quarantine for 14 days by people with mild symptoms remains most important public health intervention, but testing of all suspected cases, symptomatic contacts of probable and suspected cases, would still be required.

Cancer patients are more likely to get COVID-19 infection as they are immunosuppressive because of cancer itself or cancer directed treatment i.e. radiotherapy, chemotherapy, immunotherapy or targeted therapy.

To contain the spread of COVID-19, government has closed public places, public transport and also imposed laws to prevent social gatherings because of which it's difficult for patient to visit Radiotherapy facility daily for the treatment. Many questions appear in front of radiation oncologist, which makes decision making more crucial as no standard guidelines exists, most important being; in which patients' radiotherapy can be avoided or delayed, dose adjustment for unplanned treatment gaps during radiation therapy, how to prioritize new cases to offer maximal benefit by starting radiotherapy early and how to manage a patient infected with COVID-19 during or before radiotherapy.

\footnotetext{
Corresponding Author:

Dr. Shashank Bansal

Department of Radiation Oncology, Dr. B Borooah Cancer Institute, Guwahati, Assam, India.

Email: darpan.vcsg@gmail.com
} 


\section{Practical approach}

\section{The ultimate dilemma to offer or avoid radiotherapy}

Patient expected to derive maximum benefit by radiotherapy should not be deprived of treatment. In cases where radiotherapy has no survival benefit and only decreases locoregional recurrence it may be avoided. Braunstein et al. and Simcock et al. [5-6] has recently published their views, mentioning site directed subset of patients in which radiotherapy can be avoided. Breast cancer patients who underwent breast conservation surgery and have DCIS (Ductal Carcinoma in Situ)/ low risk invasive disease in older patients/ invasive disease with low risk genomic profile or underwent mastectomy and have T1-2, N1 disease, radiotherapy can be omitted in this group of patients. Prophylactic cranial irradiation can be omitted in small cell lung cancer patients or in patients of non-small cell lung cancer with asymptomatic brain metastasis, whole brain RT can be omitted. Low risk and favorable intermediate risk prostate cancer patients can be kept on active surveillance and radiotherapy can be omitted in oligometastatic disease. Resectable/unresectable stomach cancers, radiotherapy can be avoided. In CNS (Central Nervous System) tumors radiotherapy can be omitted in, old age patient ( $>60$ years) methylated Glioblastoma, Low grade Glioma and in asymptomatic Grade 1-2 Meningiomas [5-6]. Although enough data exists for avoiding radiation in these subsets of patients mentioned above, a careful discussion in multidisciplinary tumor board discussion is recommended.

\section{Can we delay radiotherapy?}

As per tumor biology, cancer with slow growth rate or long tumor doubling time, treatment can be delayed without fear of disease getting upstaged. As published by Simcock et al and Braunstein et al, radiotherapy can be delayed in following subset of patients; breast cancer patients who underwent breast conservation and are stage T1-2, N0, luminal A+B, can wait for radiotherapy for up to 12-20 weeks after chemotherapy. In CNS tumors, radiotherapy patients with asymptomatic meningioma WHO grade1, post-op gross total resection and WHO grade 1-2. Prostate cancer patients with unfavorable intermediate, high, very high-risk patients' radiotherapy can be delayed up to 6-7 months by using neoadjuvant hormonal therapy. Basal cell carcinoma of skin can wait for radiotherapy as it is slow growing. In palliative settings, painful metastases without impending structural/ neurological compromise can be treated with pain medications and radiotherapy can be delayed [5-6].

\section{Managing treatment gap}

Due to lockdown in various countries globally, it's not possible for one to receive radiotherapy daily or sometimes for a longer interval beyond 7 days. Patients may be unable to receive some fractions in between treatment resulting in prolongation of treatment time or may discontinue their treatment before the completion of prescribed radiotherapy dose. In these patients after weighing risk and benefit of delivering remaining radiation they may be advised accordingly. The paper by Gay et al. [6] evaluating the impact of hurricane Maria in Puerto Rico in 2017, provides guidelines on how to handle treatment interruptions for common disease sites and can be used effectively in present scenario. In breast patients the impact of gap is usually low and patients can be successfully treated with the intended dose as planned earlier. In prostate cancer patients the impact of treatment gap is very low and can be successfully taken for completion of planned radiotherapy dose after a gap. In Head and neck, Uterine Cervix, Small cell and Non-small cell Lung cancer the impact of radiotherapy gap is usually high and treatment should be resumed a soon as possible, and the gap duration should be taken in account while re-calculating the dose to be prescribed [6]. Gay et al. has described site wise treatment approach after treatment gap and we advise to go through the detail paper for those interested [7].

\section{Prioritizing radiotherapy treatment}

With the available literature it may be easier to segregate patients in two groups; first, requiring urgent radiotherapy, second, radiotherapy can be avoided or delayed. Radical radiotherapy or chemoradiotherapy with curative intent should be started in patients having rapidly proliferating tumor or treatment has already been started and the impact of treatment gap is very high. It is important to ensure that these patients complete the radiation by providing indoor admissions, dedicated patient quarters or lodge or by providing pick up and drop facility from the place of staying wherever possible. Short course radiotherapy (e.g. rectal cancers) [8] or hypo fractionated radiotherapy protocols (e.g. breast, prostate cancers, Larynx) [9-10-11] should be considered. Overall treatment time reduction without compromising the results may also help in accommodating more patients in a short span of time.

\section{Use of alternative options}

Patients in whom radiation is avoided or delayed should be considered for alternative treatment options. Various alternative cancer directed therapy like chemotherapy, hormonal therapy, immunotherapy may be utilized to give benefits to patients. Use of metronomic chemotherapy may be beneficial in patients waiting to be operated or waiting for radiotherapy [12].

\section{Treating COVID-19 positive or suspected cases}

WHO has issued guidelines for prevention of spread of COVID-19, hence Priority should be given for recovery from COVID-19, by keeping the patient in isolation for necessary period and radiotherapy should be considered as soon patient turns negative from COVID-19 [4]. Radiotherapy treatment without increasing risk of spread is possible if we judiciously categorize patients. Patients with infective symptoms, but tested negative for COVID-19, may be allowed to continue treatment with adequate protective and safety measures. In patients with suspected or proven COVID-19 infection and who are symptomatic treatment may be deferred until resolution or till they are deemed non-contagious by local health bodies. Patients 
with suspected or proven COVID-19 infection but who are asymptomatic may also be deferred treatment until their resolution or till they are deemed non-contagious by local health bodies. In selected patients (successfully treated or asymptomatic) requiring prompt initiation or continuation of radiotherapy, treatment may be allowed after observing all the necessary precautions. A careful evaluation of general health and tolerability of patients for radiotherapy after COVID- 19 infection resolution is must in all cases.

\section{Patient and relatives}

Cancer still exists as a major fear amongst Indian population. It is very much emotional and psychological burden for the patients and family members. It is very pertinent to sit together and discuss the pros and cons of avoiding and delaying the radiotherapy and its overall impact on disease control and survival with patients and their family members. In scenarios, where patients relative persuade to start treatment irrespective of benefit it carries, creates an unwanted pressure towards medical resources and care givers in hospital. In present crisis taking help of psychologists and counselors should be considered in these situations and use of electronic media like telephone or video conferencing can be considered if required.

\section{Protection of Radiation oncologists and Staff}

Fear of contacting the infection and chances of being caught into a medicolegal controversy during management of these cases are highly possible. Although many guidelines and advisory are [13-14] available for the use of PPE's (Personal Protection Equipment) and safety measures, the psychological aspect of caregivers is often overlooked. Support system of care givers is equally important as that of patients. Medical professional liability insurance of health professional should be considered either individually or through hospital.

\section{Guidelines}

Although no set guidelines are available for radiation therapy in COVID-19 pandemic scenario, we have tried to set a practical protocol-based approach. The practical flow chart (Figure 1) may be used to consider radiotherapy in this present crisis. Although this is just a suggestion a careful consideration of patient's disease, COVID status, co morbidities and support of other medical specialities including medical, renal cardiac experts should be taken by the multidisciplinary team before taking a final call.

In conclusion, our Practical flow chart may provide a basic algorithm for categorising patients, depending on various malignancy parameters and help in prioritising radiotherapy treatment in cancer patients, at present scenario of COVID-19 pandemic. Cancer centres are advised to consider these options after careful evaluation of every case and as per expertise available at each centre.

\section{Acknowledgements}

We would like to acknowledge Dr. Deepika Agrawal and Dr. Anil Matthew for their constant support and motivation. We would also like to thank the staff of our radiotherapy department for continuously providing

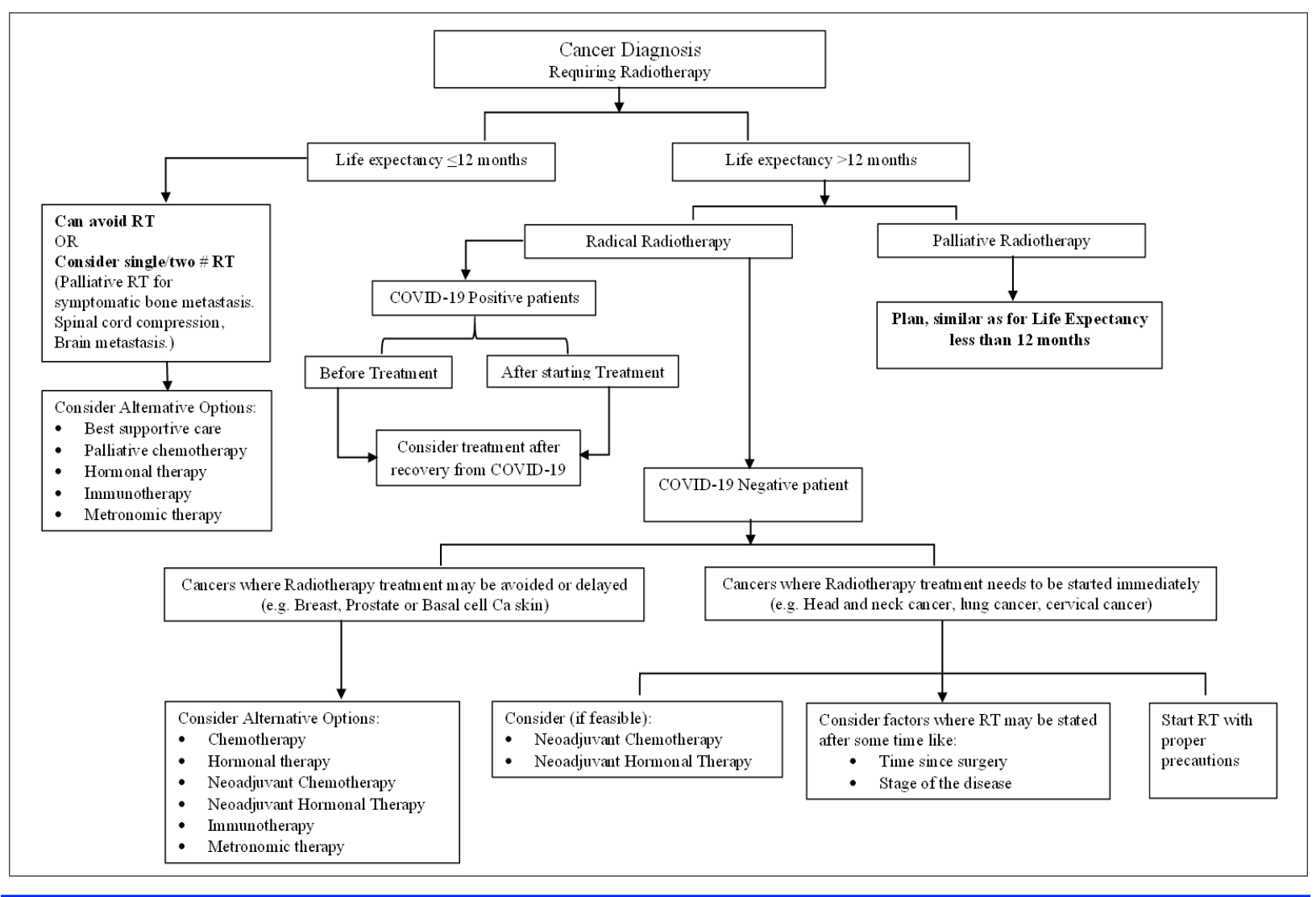

Figure 1. Practical Flowchart 
services in the situation of pandemic.

\section{Funding Statement}

No Financial support has received by any author.

\section{References}

1. WHO | Novel Coronavirus - China [Internet]. WHO. World Health Organization; [cited 2020 Apr 4]. Available from: http://www.who.int/csr/don/12-january-2020-novelcoronavirus-china/en/.

2. Coronavirus [Internet]. [cited 2020 Apr 5]. Available from: https://www.who.int/emergencies/diseases/novelcoronavirus-2019..

3. Huang C, Wang Y, Li X, Ren L, Zhao J, Hu Y, Zhang L, Fan G, Xu J, Gu X, Cheng Z, Yu T, Xia J, Wei Y, Wu W, Xie X, Yin W, Li H, Liu M, Xiao Y, Gao H, Guo L, Xie J, Wang G, Jiang R, Gao Z, Jin Q, Wang J, Cao B. Clinical features of patients infected with 2019 novel coronavirus in Wuhan, China. The Lancet. 2020 02;395(10223):497-506. https:// doi.org/10.1016/s0140-6736(20)30183-5

4. Q\&A on coronaviruses (COVID-19) [Internet] . [cited 2020 Apr 4]. Available from: https://www.who.int/news-room/qa-detail/q-a-coronaviruses..

5. Simcock R, Thomas TV, Estes C, Filippi AR, Katz MS, Pereira IJ, Saeed H. COVID-19: Global radiation oncology's targeted response for pandemic preparedness. Clinical and Translational Radiation Oncology. 2020 05;22:55-68. https://doi.org/10.1016/j.ctro.2020.03.009

6. Braunstein LZ, Gillespie EF, Hong L, Xu A, Bakhoum SF, Cuaron J, Mueller B, McCormick B, Cahlon O, Powell S, Khan AJ. Breast Radiation Therapy Under COVID-19 Pandemic Resource Constraints-Approaches to Defer or Shorten Treatment From a Comprehensive Cancer Center in the United States. Advances in Radiation Oncology. 2020 04;. https://doi.org/10.1016/j.adro.2020.03.013

7. Gay HA, Santiago R, Gil B, Remedios C, Montes PJ, LópezAraujo J, Chévere CM, Imbert WS, White J, Arthur DW, Horton JK, Jagsi R, Rabinovich R, Beriwal S, Viswanathan A, Erickson BA, Rengan R, Palma D, Loo BW, Kavanaugh JA, Bradley J, Yom SS, Harari PM, Lee Burnett O. Lessons Learned From Hurricane Maria in Puerto Rico: Practical Measures to Mitigate the Impact of a Catastrophic Natural Disaster on Radiation Oncology Patients. Practical Radiation Oncology. 2019 09;9(5):305-321. https://doi.org/10.1016/j. prro.2019.03.007

8. Wang X, Zheng B, Lu X, Bai R, Feng L, Wang Q, Zhao Y, He $\mathrm{S}$. Preoperative short-course radiotherapy and long-course radiochemotherapy for locally advanced rectal cancer: Metaanalysis with trial sequential analysis of long-term survival data. Batra SK. PLOS ONE. 201807 12;13(7):e0200142. https://doi.org/10.1371/journal.pone.0200142

9. The UK Standardisation of Breast Radiotherapy (START) Trial B of radiotherapy hypofractionation for treatment of early breast cancer: a randomised trial. The Lancet. 2008 03;371(9618):1098-1107. https://doi.org/10.1016/s01406736(08)60348-7

10. Dearnaley D, Syndikus I, Mossop H, Khoo V, Birtle A, Bloomfield D, Graham J, Kirkbride P, Logue J, Malik Z, Money-Kyrle J, O'Sullivan JM, Panades M, Parker C, Patterson H, Scrase C, Staffurth J, Stockdale A, Tremlett J, Bidmead M, Mayles H, Naismith O, South C, Gao A, Cruickshank C, Hassan S, Pugh J, Griffin C, Hall E. Conventional versus hypofractionated high-dose intensitymodulated radiotherapy for prostate cancer: 5 -year outcomes of the randomised, non-inferiority, phase 3 CHHiP trial.
The Lancet Oncology. 2016 08;17(8):1047-1060. https:// doi.org/10.1016/s1470-2045(16)30102-4

11. Bledsoe TJ, Park HS, Stahl JM, Yarbrough WG, Burtness BA, Decker RH, Husain ZA. Hypofractionated Radiotherapy for Patients with Early-Stage Glottic Cancer: Patterns of Care and Survival. JNCI: Journal of the National Cancer Institute. 201704 26;109(10). https://doi.org/10.1093/jnci/djx042

12. Prabhash K, Noronha V, Krishna M, Patil V, Joshi A, Banavali S. Metronomic therapy: Chemotherapy revisited. Indian Journal of Cancer. 2013;50(2):142. https://doi. org/10.4103/0019-509x.117027

13. Health Workers [Internet]. [cited 2020 Apr 4]. Available from: https://www.who.int/emergencies/diseases/novelcoronavirus-2019/technical-guidance/health-workers..

14. The COVID-19 pandemic and the Tata Memorial Centre response. Indian Journal of Cancer. 2020;0(0):0. https://doi. org/10.4103/ijc.ijc_250_20

\section{(i) (3)}

This work is licensed under a Creative Commons AttributionNon Commercial 4.0 International License. 